\title{
An Ontology-based Knowledge Framework for Engineering Material
}

\author{
Selection \\ YingzhongZhang $^{\mathrm{a}^{*}}$, XiaofangLuo $^{\mathrm{a}}$, YongZhao $^{\mathrm{a}}$, Hong-chaoZhang $^{\mathrm{b}}$ \\ ${ }^{a}$ School of Mechanical Engineering, DalianUniversity of Technology, Dalian, \\ 116024, China \\ ${ }^{\mathbf{b}}$ Department of Industrial Engineering, Texas Tech University, Lubbock, TX 79409, \\ USA
}

Corresponding author name: Yingzhong Zhang

Affiliation: School of Mechanical Engineering, Dalian University of Technology

Detailed address: School of Mechanical Engineering, Dalian University of

Technology, Dalian, China, 116024

Email: zhangyz@dlut.edu.cn

Tel: $+\mathbf{8 6 - 0 4 1 1 - 8 4 7 0 8 6 1 4}$

Fax: +86-0411-84708614

*Corresponding author.Tel.: +86411 84708614; fax: +8641184708614.

E-mail address: zhangyz@dlut.edu.cn 


\title{
An Ontology-based Knowledge Framework for Engineering Material
}

\author{
Selection \\ YingzhongZhang ${ }^{\mathrm{a}^{*}}$, XiaofangLuo $^{\mathrm{a}}$, Yong Zhao ${ }^{\mathrm{a}}$, Hong-chaoZhang ${ }^{\mathrm{b}}$

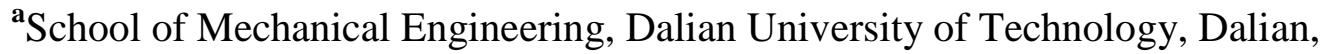 \\ 116024, China \\ ${ }^{\mathbf{b}}$ Department of Industrial Engineering, Texas Tech University, Lubbock, TX 79409, \\ USA
}

Abstract:Engineering material selection intensively depends on domain knowledge. In the face of the large number and wide variety of engineering materials, it is very necessary to research and develop an open, shared, and scalable knowledge framework for implementing domain-oriented and knowledge-based material selection. In this paper, the fundamental concepts and relationships involved in all aspects of material selection are analyzed in detail. A novel ontology-based knowledge framework is presented. The ontology-based Semantic Web technology is introduced into the semantic representation of material selection knowledge. The implicit material selection knowledge is represented as a set of labeled instances and RDF instance graphs in terms of the concept model, which provides a formal approach to organizing the captured material selection knowledge. A knowledge retrieval and reasoning approach integrating ontology concepts, instances, knowledge rules, and semantic queries encoded with Query-enhanced Web Rule Language (SQWRL) is proposed. The presented knowledge framework can provide powerful knowledge services for material selection. Finally, based on this knowledge

\footnotetext{
"Corresponding author.Tel.: +86411 84708614; fax: +8641184708614.

E-mail address: zhangyz@dlut.edu.cn
} 
framework, a case study on constructing a mold material selection knowledge system is provided. This work is a new attempt to build an open and shared knowledge framework for engineering material selection.

Keywords: Engineering materials; Material selection; Knowledge representation; Ontology

\section{Introduction}

With the development of technologies, the available set of engineering materials is rapidly growing in both type and number. It is estimated that there are more than 80,000 engineering materials available in the world, and new materials are emerging constantly [1]. Engineering materials are usually coupled with series of manufacturing processes. It is estimated that there are at least 1000 different manufacturing processes that can convert engineering materials into desired products[1]. In the material selection process, design engineers have to take into account a large number of factors, such as physical properties, mechanical properties, thermal properties, material cost, and impact on the environment. Hence, the vast number of materials and processes and the complex relationships between the different selection parameters often make the selection of materials for a given component a difficult task [2].

Information on engineering materials presents in two categories: data and knowledge. Data is defined as the result of measurements that can be presented as numerical values, whereas knowledge represents the connections between the items of data [3].As the materials involve a large amount of data, it is necessary to employ 
database information systems to effectively manage, retrieve, and update the material data. A large number of material databases have been built, most of which can be accessed online [4]. Due to their data-oriented representation mode, the material databases still lack a knowledge inference mechanism and are not able to associate the data with facts.

Material selection is a highly knowledge-intensive activity, involving knowledge mapping among different concepts, knowledge inference, and multiple objective decision-making. Hence, efficient mechanisms for capturing, representing, reusing, and sharing the material selection knowledge involved are sorely needed. As a result, much research on knowledge-based engineering material selection tools and methods has been carried out. However, many challenges in knowledge-based material selectionstill exist, as in any other knowledge-based engineering [5]. One challenge is that the material selection knowledge is implicit and unstructured; therefore, it is very difficult to capture the material selection knowledge and give it formal representation. Another challenge is that material selection depends on applications in different domains; therefore, an open, scalable, and shared knowledge framework is required. Based on this knowledge framework, the material selection knowledge base for different domains can be effectively constructed and updated.

Recently, knowledge representation technology based on ontologies and Semantic Web technologies have received more attention and been gradually used in the knowledge representation of various domains. An ontologycan effectively combine the domain knowledge with the information representation andhas many advantages 
over other concept modeling technologies in classification, sharing, and formalization [6-9]. The ontology technology provides a feasible solution to addressing the issues and challenges.

With this in mind, this paper analyzes the knowledge requirements for material selection and discusses the common characteristics and relationships among the engineering materials, manufacturing processes and applications. A novel ontology-based knowledge framework for engineering material selection is proposed that includes three layers, namely, the knowledge base layer, the reasoning layer, and the user interface layer. The main contributions of this paper include (i) an ontology-based concept model for representing material selection knowledge, (ii) an approach to representing implicit material selection knowledge by knowledge instances and rules, and (iii) an approach to semantically retrieving material selection knowledge and screening desired materials from the knowledge base. The presented work will lay the foundation for constructing an open, shared, and scalable material selection knowledge framework.

\section{Literature review}

As mentioned above, due to the importance of material selection in engineering design, material selection tools and methods have been extensively researched. Details can be found in [3]. Here, we limit our review of previous works to those discussing knowledge-based material selection. 


\subsection{Knowledge-based material selection}

A knowledge-based system (KBS) is a computer program that reasons and uses a knowledge base to solve complex problems. In recent decades, knowledge-based material selection methods attracted much research. Goel and Chen [10] early employed an expert network for material selection inengineering design. Sapuan and Abdalla[11] presented a prototype knowledge-based system for the selection of polymer-based composite materials for a pedal box system of an automotive. Sanyang and Sapuan[12] developed an expert system using ExsysCorvid software to select suitable biobased polymer materials for packaging products. In their developed expert system, an "If-Then" rule is utilized in the material selection process, whereas a scoring system was formulated to facilitate the ranking of the selected materials. Zarandi et al. [13] proposed a material selection methodology and an expert system for sustainable product design. Ipek et al. [14] proposed an expert system based material selection approach to manufacturing. Urrea et al. [15] developed an expert system for the selection of materials to be used in the construction of the main structure of a transfer crane. In their approaches, the expert system determines the appropriate material properties for the product design and then searches for appropriate materials having these properties in the materials knowledge base. Zha[16] described the selection of suitable manufacturing processes and materials in concurrent design according to a fuzzy knowledge. Based on the proposed method, a prototype web-based knowledge-intensive manufacturing consulting service system was developed. Kumar and Singh [17] presented an intelligent system for the 
selection of materials for progressive die components. Amen and Vomacka[18] used case-based reasoning $(\mathrm{CBR})$ as a tool for material selection. CBR is a procedure of solving new problems based on the solutions of similar past problems. Ullah and Harib[19] presented an intelligent method for selecting optimal materials. The presented method uses material properties instead of production rules and inference calculations, but its application scope is limited.

From the published literature, the following issues exist in the current expert systems and knowledge systems for material selection:

(1) The material selection knowledge is mainly represented as a set of general "IF-THEN" production rules. Few systems can comprehensively use concepts, instances, and rules to represent the complex material selection knowledge, which is implicit and unstructured. The knowledge instance created from material selection and applications is an important knowledge representation form.

(2) The research on semantic representation for material selection knowledge at the conceptual level is generally lacking. For example, the variables in the production rules are not defined from shared concepts and closely coupled with the application program; therefore, it is difficult to modify variables or add new variables to the rule base. As a result, such an expert system for material selection is often limited to a specific application domain. It is necessary to define a set of shared knowledge concepts and use these concepts in the representation of knowledge rules and instances. 
(3) Current material selection systems commonly do not use open knowledge processing platforms. An open knowledge modeling platform (including a modeling language, inference engine, and developing tools) can promote the development and application of the material selection knowledge system.

\subsection{Ontology-based knowledge systems for material selection}

According to Gruber [20], an ontology is an explicit specification of a conceptualization. Borst[21] further specified the definition of an ontology as a formal specification of a shared conceptualization. The two definitions indicate that the ontology is a concept representation of an abstract domain and is understood and shared among human and machines (software agents). The ontology and Semantic Web technologies provide powerful reasoning capabilities; therefore, ontology-based modeling has many advantages over other concept modeling technologies in classification, sharing, and formalization. Ontologies have been extensively used to formalize domain knowledge with concepts, attributes, relationships, and instances in many scientific and industrial fields. There is a tendency to both convert existing models into ontologies and to create new ontology-based models. For example, Zhan et al. [22] presented a semantic approach that uses ontologies to share knowledge related to product data in CAD/CAE applications. Zhang et al. [23] presented an ontology-based knowledge representation for unit manufacturing processes. Matsokis and Kiritsis[24] developed an ontology-based approach for product lifecycle management. Sanya and Shehab[25] developed an ontology framework for 
developing platform-independent knowledge-based engineering systems in the aerospace industry.

Some efforts have been made in engineering material information representation and processing. The Materials Markup Language (MatML) [26] made efforts to create a materials information exchange standard based on XML schemas. However, due to the lack of semantics in XML representation, it is difficult to realize the goal of the material information exchange. Ashino[27] proposed a material ontology that describes concepts in materials science and engineering and the relationships among them. The proposed material ontology has been applied to exchange data among three different thermal property databases. Rahmani et al. [28] presented an ontology-based integration of heterogeneous material data resources from different stages of the product lifecycle. Cheng et al. [29] presented a semantic-driven knowledge representation model for materials engineering applications. Their works aim at the semantic representation of engineering material data, service reasoning, and application evaluation. From the published literature, considerably fewer works have been involved in ontology-based material selection. Ashino and Fujita [30] defined a Web ontology for design-oriented material selection. They considered that the material selection for designing artifacts is a process that translates required material properties into material substances and proposed an ontological structure to formalize this process. However, the presented work is still preliminary and does not provide many details. Liu et al. [31] presented a fuzzy matchmaking approach for Semantic Web Services for collaborative material selection. Their works mainly aim at 
addressing the problem of service matching and discovery in the Web service. Premkumar et al. [32] developed a novel Semantic LaminatedComposites Knowledge Management System (SLACKS) that is based on a suite of ontologies for laminatedcomposite materials. SLACKS aims at creating a semantic knowledge structure for laminated composite product design and the standardization of concepts in the laminated composite domain. However, it mainly focuses on knowledge integration for relevant domains of the laminated composite product life cycle, such as design, analysis, manufacturing, and material selection.

In summary, we can see that many challenges and issues in knowledge-based material selection have not yet been addressed. It is necessary to research the knowledge frame for material selection by making full use of ontology and Semantic Web technologies.

\section{Overview of the framework}

To cope with the issues and challenges discussed above, the material selection knowledge framework design should consider at least the following three aspects:

(1) Material selection knowledge representation.

A knowledge representation is a machine-interpretable representation of the real world, in which symbols serve as surrogates for real world domain artifactsand can be used to conduct reasoning for the domain of interest [33]. Engineering material information has a number of unusual characteristics compared with information from other engineering domains. One uncommon characteristic is open-endedness 
[34].Materials information is a thin thread that runs through many different engineering activities, including design, manufacture, maintenance, and disposal. In addition, it implies that any materials knowledge system will never be complete; there will always be relevant knowledge that it does not contain [34]. Hence, an open and shared knowledge representation mode and a knowledge concept model thatcan represent all aspects involved in material selection are needed.

(2) Effective knowledge services for material selection.

The knowledge framework should provide powerful knowledge reasoning capabilities. Based on the knowledge base, the framework can semantically reason and retrieve the candidate materials according to different requirements, such as design requirements and manufacturing conditions. In addition, for a specific product, multiple objective attributes for material selection and their relative importance can be retrieved and integrated into other multiple objective decision-making methods.

(3) Knowledge base construction mode and tools.

The knowledge base is the key to a knowledge system. In general, the knowledge base stores the symbols of the computational model in the form of statements about the domain. How to effectively describe and organize the knowledge statements is a fundamental issue in a knowledge system. In addition, abundant software tools for constructing a knowledge base can promote the application of the knowledge system.

According to the above analysis, this paper proposes an ontology-based knowledge framework for engineering material selection, as shown in Fig. 1, which 
consists of the following three layers, namely, the knowledge base layer, the reasoning layer, and the user interface layer.

(1) Knowledge base layer.

Currently, W3C has finalized Web Ontology Language (OWL) as the modeling language [35] and Semantics Web Rule Language (SWRL) as the rule language to implement ontology technologies [36]. The combination of both languages makes OWL have more powerful knowledge representation and reasoning capabilities. Therefore, the knowledge base layer is constructed on OWL DL, which includes a terminology box (TBox), an assertion box (ABox) and a rule base. The TBox defines the terminology, i.e., the concepts, axioms, and relationships between concepts in the engineering material domain. The ABox contains the knowledge instances in terms of concepts, in which the knowledge instances are organized according to a Resource Description Framework (RDF) graph. In addition, although OWL supports the inference, it does not provide the reasoning rules such as "IF... THEN..."; therefore, its reasoning ability is very limited. Hence, a rule base encoded with the SWRL language is defined in the knowledge base.

(2) Reasoning layer.

The reasoning layer mainly performs ontology reasoning tasks. Currently, Semantic Query-enhanced Web Rule Language (SQWRL) [37], an SWRL-based query language, has been widely used. SQWRL takes a standard SWRL rule antecedent and effectively treats it as a pattern specification for a query. An implementation of SQWRL has been developed in the SWRLTab plugin [37] in 
Protégé-OWL (http://plugins/owl/api/protege.stanford.edu). Hence, in the reasoning layer, SQWRL is employed as the query language, and the Protégé-OWL ontology development toolkit supporting Jess is adopted as a software implementation tool[38]. The interface implementation between the querying applications and the SQWRL engine can be found in[23].

(3) User interface layer.

The user interface layer provides two interfaces. One interface is used for constructing the knowledge base, which is also called the knowledge constructor, consisting of two modules. One module defines the knowledge concepts and instances and is carried out in Protégé 4.3 editor(http://www.protege.stanford.edu). Another module is a knowledge rules editor developed by the authors. In the user interface layer,under the guidance of the ontology model, the material selection knowledge related to a specific domain is captured, abstracted and instantiated. The constructed knowledge base is saved in a "materials.owl" file that is stored in a shared network namespace.

The other interface is used for querying or retrieving engineering material information, which is also called the material selector. The material selector is an application program for selecting suitable materials, which is developed on Eclipse Java development tools (http://www.eclipse.org). The material selector first converts the request for material selection into SQWRL querying statements, submits the statements to the reasoning layer mentioned above, and then processes and shows the 
retrieval results. Other multiple attribute or criterion decision-making methods can also be integrated into this material selector.

The material selection knowledge representation is the most crucial part of the knowledge framework, which is elaborated as follows.

\section{An ontology-based concept model for engineering material selection}

\subsection{Conceptual analysis of the material selection knowledge}

The objective of material selection is to select the most suitable material from the set of available materials to ensure that a product made of the selected material can meet the design requirements. On the one hand, the material selection knowledge involves all aspects from the material itself to the material processing and application. On the other hand, the material selection knowledge usually exists in the various implicit relationships related to a specific domain and is difficult to structure. Hence, how to describe and represent the unstructured and implicit material selection knowledge is a crucial issue.

In general, a concept is an abstract form to represent entities and relationships. The content (meaning) of the concept includes all of the properties and relations of the concept. Knowledge can be described as some interconnected concepts, and each concept is connected through associations[39]. Hence, concepts are the foundation for knowledge representation. The material selection knowledge mainly involves the following concepts. 
(1) Engineering material concepts.

Engineering material concepts represent the most essential and common characteristics of engineering materials, which can be described using two types of attributes. One is the description of the material nature, including various physical and chemical properties, chemical composition, and material microstructure. The other is the associated relationships between materials and manufacturing processes, product applications, and so on. The attributes of an engineering material can be defined as

$$
A=\left\{a_{1}^{p}, a_{2}^{p}, a_{3}^{p}, \ldots, a_{m}^{p}, a_{1}^{r}, a_{2}^{r}, a_{3}^{r}, \ldots, a_{n}^{r}\right\}
$$

where $m$ is the number of material properties, and $n$ is the number of associated relationships.

(2) Manufacturing process concepts.

A manufacturing process describes the manufacturing methods by which an engineering material is made into a product, usually including processes of shaping, joining, or finishing materials. A material is chosen because it is capable of being processed to the shape and quality required by the design requirements. The choices of materials and processes are tightly coupled [1].

(3) Engineering product concepts.

A physical product is made of one or more materials. A product design proposes various technological requirements for materials. In long-term practice, the application relationships between materials and products for a specific domain have been established, which constitute a form of important material selection knowledge.

(4) Cost and ecological concepts. 
An important factor in material selection is to minimize the material cost and the impacts on the environment and resources. The material cost should include the cost of the material itself, processing costs, and usage costs.

\subsection{Anontology-based concept model for material selection}

Based on the above concept analysis, this paper proposes an ontology-based concept model for representing material selection knowledge, as shown in Fig. 2. On the one hand, the product design puts forward design requirements, including materials, shape, quality, and cost. On the other hand, an engineering material has specific material properties and a material cost and needs to experience a set of manufacturing processes by which it is converted into the desired product. In addition, the production of raw materials and the product manufacture consume natural resources and produce emissions to the environment. The following will elaborate how the concept model represents the material selection knowledge.

\subsubsection{Engineering material concepts}

In this paper, theEngMaterial class is defined to represent the engineering material concept. The definition of the material concept considers the following two aspects:

(1) Taxonomy of engineering materials.

In the face of the vast number and wide variety of engineering materials, the taxonomy of engineering materials is an important knowledge activity. First, we define two subclasses of the EngMaterial class: EngProduct class and RoughMaterialclass. Currently, the most common taxonomy classifies engineering 
materials according to their chemical composition because the composition and microstructure of a material determine its various properties. Hence, the RoughMaterial class derives its four subclasses: Composite class, Polymer class, Metal class and Ceramic class, and each subclass can also continue to derive its subclasses. The EngProduct class represents the materials that have been processed. The hierarchy of EngMaterial class is shown in Fig. 3 (a).

(2) Properties of the engineering material class.

In general, the meaningof the conceptis represented by its properties. In addition to the above hierarchical relationship definitions, the main properties of the EngMaterialclass are defined as shown in Fig. 2.

\subsubsection{Material property concepts}

The material property is any characteristic "behavior" of the material. In this paper, the MatProperty class is defined to describe all material property concepts. According to the common characteristics, the material property concepts and their hierarchical relationships are defined as shown in Fig. 3(b).

In general, the material properties reflect the inherent performance properties and environmental properties of materials, which are usually represented as a set of property concepts associating numerical data obtained by measurements at some conditions and a measurement unit. In representing material properties, a wide variety of units of measurement may be involved. Hence, we employ the MeasureUnit class to represent the unit of measurement of the material properties, as shown in Fig. 4. 
In Fig. 4, the MeasureUnit class is defined as a base class and derives many practical subclasses of the measurement unit. These measurement unit concepts can be automatically transformed between units by the transformation factor.

\subsubsection{Manufacturing processes and materials}

A manufacturing process is a method of shaping, joining, and finishing materials. Currently, there are hundreds of commercially used processes, including casting, injection molding, welding, machining, and electro-polishing. Different materials are suitable for different manufacturing processes. For instance, cast iron materials are usually manufactured into box parts by casting processes. Processing can also change some properties of the material. For example, a proper heat treatment process can improve some properties of the material. The relationships between materials and processes constitute a part of the material selection knowledge.

This paper defines the MfgProcess class to represent all of the manufacturing process concepts. According to the manufacturing characteristics of processing materials, the hierarchical relationship of the MfgProcess class is as shown in Fig. 3 (c). The manufacturing process is a complex concept. This paper discusses only some of its aspects relating to material selection; the relationships between the manufacturing process concepts and other related concepts are illustrated in Fig. 5. 


\subsubsection{Design requirements for engineering materials}

In general, material selection usually starts from meeting the product's design requirements for materials [2]. The design requirements are the main sources for determining the criteria of material selection, which mainly include function requirements, performance requirements, manufacturing requirements, cost requirements, and environmental requirements. We divide the design requirements into two categories, constraint requirements for material properties and objective requirements for ranking candidate materials. The objective requirements are usually affected by a group of material attributes or criteria. For example, the "cost" requirement is affected by the material cost, the manufacturing cost and the use cost, and each cost item has a different influence weight on the total cost requirement. Hence, we design an InfluenceAttributeclass and a hasAttribute property for the ObjectiveRequirement class. The hasAttribute property associates the ObjectiveRequirement class with the InfluenceAttributeclass. The DesignRequirement class and its properties are defined as shown in Fig. 6.

\section{Knowledge instances and rules for material selection}

The above ontology model only provides a semantic representation for material selection knowledge at the conceptual level. In practice, material selection knowledge is implicit and unstructured. At the same time, the engineering materials, manufacturing processes and product applications are each composed of many individuals. The material selection knowledge is usually implied in these specific 
individuals. Hence, we can build up a material selection knowledge base by organizing and describing these individuals in terms of the presented concept model.

The ontologies defined above explicitly provide hierarchical semantic relationships for concepts of the material selection knowledge. However, a large number of causal relationshipsstill exist,which cannot be represented by the OWL ontology alone. The production rule is an effective method to represent the causal relation knowledge and has been widely applied to various types of knowledge systems. It is very necessary to combine the ontological concepts and instances with production rules. The formalized material selection knowledge rules can enrich the deductive reasoning capabilities. The following will elaborate upon the construction of the knowledge instance base and rule base.

\subsection{Material selection knowledge instances}

As is well known, each specific material has specific mechanical, physical, and chemical properties, processing methods, and environmental impacts. Each specific individual is known as an instance. In this paper, all of the individuals that are used to describe the material selection knowledge are defined as material knowledge instances. The material knowledge instances and relationships between them constitute the essential domain knowledge for material selection. The following will discuss how to represent and construct the material knowledge instances at the following three levels:

(1) A single material knowledge instance. 
In the ontology-based semantic representation, an instance is a specific realization of an ontology class. The creation of a realized instance is called instantiation. The relationship between an instance and an ontology class is declared by using rdf:type in RDF. For example, Alloy 6061 is one of the most common aluminum alloys; therefore, it can be defined as an instance of the 6000Series class.

(2) A material knowledge instance graph.

The material properties of a specific material should be relatively stable, but it can be converted into different products through different manufacturing processes, which means that there are multiple different combinations of knowledge instances. Each combination of material knowledge instances can be described as an RDF graph, in which a node denotes an instance of an ontology class in TBox and an edge denotes a relation between instances. In this paper, a complete material selection relationship chain from rough materials to manufacturing processes, final products, and design requirements is defined as a material selection. Fig. 7 illustrates a material selection of a car hub product with an RDF graph representation. "6061" is an instance of the 6000Series class, and "Hub_1" is an instance of the CarHub class.

(3) A material knowledge instance base.

All of the defined material selection instances constitute an instance base that is also known as the ABox of the material knowledge base. The ABox for material selection knowledge can be represented as

$$
\mathrm{ABox}=\left\{M_{1}^{1}, M_{1}^{2}, \cdots, M_{1}^{k}, \cdots, M_{m}^{1}, M_{m}^{2} \cdots, M_{m}^{n}\right\} .
$$


In the above expression, $M_{i}$ denotes a material selection, and $m$ denotes the number of material selections in the ABox; $k$ denotes the number of instances in material selection $M_{1}$, and $n$ denotes the number of instances in material selection $M_{m}$.

To infer the correct results, each instance in ABox is required to undergo tests of consistency and completeness regarding its related concepts. A consistent knowledge base is free of conflicts and has identical and subsumed knowledge instances and rules. The tests of the consistency and completeness are to verify and remove conflicting instances, redundant instances, and incomplete instances. For example, an instance of the EngMaterial class is only allowed to connect to an instance of the MfgProcess class through the "inputs" property. Similarly, if an instance of the EngMaterial class does not connect to any instance of the MfgProcess class through the "inputs" property, the instance is considered incomplete.

\subsection{Knowledge rules for material selection}

\subsubsection{Knowledge rules encoded with SWRL}

As mentioned above, it is very necessary to combine the ontological concepts and instances with production rules to construct the knowledge rules. On the one hand, the relationships in OWL ontologies can be represented as a set of description logic (DL) predicates, which gives the relations precise semantics. In DL, concepts are mapped to unary and relations to binary predicates. The meaning of a variable in a relation expression is determined by the domain and range defined in the ontology. On the other hand, SWRL is based on the combination of the OWL DL and RuleML[36]; 
therefore, it can effectively combine ontological concepts and instances with production rules. Hence, in this paper, SWRL is chosen to define and encode the knowledge rules.

An SWRL rule contains an antecedent part, which is referred to as the body, and the consequent part, which is referred to as the head[36]. An SWRL reasoning rule can be expressed in the form:Antecedent $\rightarrow$ Consequent, and the antecedent part and the consequent part can also be expressed as the conjunctive formula of atoms $a_{1}^{\wedge} a_{2} \wedge, \ldots, \wedge a_{\mathrm{n}}$ and $b_{1} \wedge b_{2}{ }^{\wedge}, \ldots,{ }^{\wedge} b_{\mathrm{m}}$, respectively. Atoms $a_{\mathrm{i}}$ and $b_{\mathrm{j}}((1 \leqslant i \leqslant n, 1 \leqslant j \leqslant m)$ can be in either of the forms $C(? x)$ or $P(? x, ? y)$, in which if $x$ is an instance of class $C$, then $C(? x)$ holds; if $x$ is related to $y$ by property $P$, then $P(? x, ? y)$ holds.

The causal relationship knowledge of material selection can be represented as knowledge rules encoded with SWRL. The following will introduce how to construct the knowledge rules from the following two aspects.

\subsubsection{Knowledge rules for mapping the concepts between design requirements and material properties}

Material selection is a process matching the design requirements with the material's various properties. However, the design requirements for materials are usually from the point of view of the product design, existing in the product design space, and the material properties are the various characteristics that a material originally possesses, which exist in the material property space. The former is macro, and the latter focuses on specific data. One item of design requirements often corresponds to several material properties. The mutual semantic mapping 
relationships are important contents of the material selection knowledge, as illustrated in Fig. 8. It is necessary to capture them and give them explicit and formal definitions in knowledge rule forms. In general, there are the following two types of mapping forms.

(1) Mapping a combination of material properties to a new material concept.

In the material selection process, sometimes a new material concept is used to represent some design requirements for materials. For instance, the design requirement for the material weight is represented as the lightweight material concept or the heavy material concept. Hence, we can implement the mapping by defining a set of new material concepts that has an intersection set of material property constraints. The knowledge rules can be formally defined as follows:

$$
\begin{gathered}
C_{1}^{1} \wedge C_{2}^{1} \wedge C_{3}^{1} \cdots C_{m}^{1} \rightarrow M_{1}, \\
\cdots \cdots, \\
C_{1}^{k} \wedge C_{2}^{k} \wedge C_{3}^{k} \cdots C_{n}^{k} \rightarrow M_{k} .
\end{gathered}
$$

In the above formula, $M$ denotes the newly defined material concepts, and $C$ denotes the material properties or constraint relations of the property value. Table 1 gives a set of knowledge rule examples encoded with SWRL.

(2) Mapping a combination of design requirements to a combination of material properties.

In practical product design, a product may propose a set of design requirements for materials, forming a combination of design requirements. Correspondingly, there is a combination of material properties that can meet the combination of design requirements. This type of causal relation knowledge can be formally defined by the 
following rules:

$$
\begin{aligned}
R_{1}^{1} \wedge R_{2}^{1} \wedge R_{3}^{1} \cdots R_{i}^{1} & \rightarrow P_{1}^{1} \wedge P_{2}^{1} \wedge P_{3}^{1} \cdots P_{m}^{1} \\
\cdots \cdots, & \\
R_{1}^{k} \wedge R_{2}^{k} \wedge R_{3}^{k} \cdots R_{j}^{k} & \rightarrow P_{1}^{k} \wedge P_{2}^{k} \wedge P_{3}^{k} \cdots P_{n}^{k} .
\end{aligned}
$$

In the above formula, $P$ denotes the material properties or constraint relations of the property value, and $R$ denotes the design requirements or constraint relations of the design requirement value. The subsequent case study will provide partial knowledge rules for mapping die casting design requirements to material properties.

\subsubsection{Knowledge rules for mapping concepts between manufacturing processes and material properties}

A manufacturing process converts raw materials into finished products with specific shape, structure, and material properties. In general, each manufacturing process requires different material properties. For instance, the casting process requires that the casting material have good heat flowability, while the forging process requires that the material have a good plasticity. We can capture the knowledge of different property requirements of the manufacturing process and define it as a set of new concepts on the material's manufacturing capability. Table 2 provides a set of knowledge rule examples to define the manufacturing capability concepts.

\section{Knowledge services for material selection}

In the material selection process, design engineers usually access knowledge 
services from various knowledge sources (e.g., experience, handbooks, and mature cases), which can help them to make material selection decisions or find new material data. This knowledge framework aims to provide much more effective knowledge services, including semantic inference and retrieval for material selection.

\subsection{Semantic retrieval using SQWRL}

Through the above steps, an ontology-based knowledge base for material selection encoded with OWL and SWRL has been built up. It is necessary to set up an interface between the designer's material selection intention and the knowledge base, by which the material selection knowledge can be retrieved from the knowledge base. As mentioned in Section 3, at the reasoning layer of our knowledge framework, SQWRL is employed as the query language. SQWRL defines a set of SQL-like query operators that can be used to construct retrieval specifications for information stored in an OWL ontology. These operators are used in the consequent of a SWRL rule, and the antecedent is effectively treated as a pattern specification for the query. The core built-in defined by SQWRL is sqwrl:select. This built-in takes one or more arguments, which are typically variables used in the antecedent of a SWRL rule.

SQWRL can effectively combine ontological concepts and properties with instances, production rules, and Structured Query Language (SQL) statements. Hence, through the query statement, the interface between the designer's material selection intention and the knowledge base can be set up. On the one hand, the material selector at the user interface layer converts the user's design requirements for materials into 
SQWRL query statements and submits them to the reasoning layer. On the other hand, in terms of the submitted query statements, the reasoning engine finds and extracts the required information from the knowledge base and material database and then returns the query results to the material selector.

\subsection{Knowledge retrieval and inference mechanism}

To provide effective knowledge services, the knowledge framework is required to have powerful semantic retrieval and inference capabilities. The following will demonstrate its retrieval and inference mechanism from two aspects.

\subsubsection{Reasoning on DLs}

DL is a family of knowledge representation languages used to represent the knowledge of a domain in a structured fashion. In addition, DL is decidable; therefore, there is an automatic reasoning procedure for every knowledge specification mechanism in the logic. DL has mechanisms to find implicit consequences based on explicit information. DL inference typically can perform four types of reasoning task [40], namely, consistency checking, subsumption, realization, and retrieving.

A knowledge base based on DLs includes a TBox containing all of the concepts, axioms, and rules of the ontology and an ABox containing all of the instances and their relationships regarding the concepts. Reasoning is then the satisfiability checking of ABox to TBox. Most OWL inference engines are logical and use tableau algorithms. As a result, their reasoning is sound and complete. 


\subsubsection{Retrieval based on SWRL rules}

As mentioned above, the antecedent part of an SQWRL query operates like a standard SWRL rule antecedent. SWRL extends the set of OWL axioms to include Horn-like rules. It thus enables Horn-like rules to be combined with an OWL knowledge base. In addition, in SQWRL, queries can also operate in conjunction with SWRL rules to retrieve knowledge inferred by those rules. The consequent of these rules constitutes an intermediate inference and can be used as an atom of the antecedent part of another SQWRL, which provides a mechanism to decompose very complex queries. For example, we have defined a lightweight material concept in Section 5; therefore, a query to list all lightweight metal materials from a knowledge base can then be written:

$\operatorname{Metal}(? m) \wedge$ LightweightMaterial $(? m) \rightarrow \operatorname{sqwrl}: \operatorname{select}(? m)$.

The intermediate inference mechanism provides a solution to mapping the concepts in different domains.

\subsection{Knowledge retrieval for product design requirements for materials}

In general, a mature product keeps a set of stable design requirements for materials. Identifying them heavily relies on the product knowledge and experience of designers. This knowledge framework can identify and retrieve the design requirements of the product to be designed from the knowledge base.

In the concept model for material selection, we set the DesignRequirement class and the EngProduct class. The EngProductclass is associated with the DesignRequirement class by the "proposes" property. Hence, the procedure for 
retrieving the design requirements starts from the product class to be designed. Then, a set of design requirement instances, $R=\left\{r_{1}, r_{2}, \ldots, r_{n}\right\}$, are retrieved by the "proposes" property. The following is the query statement:

MyProductClass $(? p) \wedge$ proposes $(? p, ? r) \wedge$ DesignRequirement $(? r) \rightarrow \operatorname{sqwrl}: \operatorname{select}(? r)$.

Finally, the ontology class of each design requirement instance and their class properties are identified. The main query statements are as follows.

$$
\begin{aligned}
& \text { abox:hasClass(? } \left.\left.r_{\mathrm{i}}, ? \text { ReqClass }\right) \rightarrow \text { sqwrl:select(?ReqClass }\right) \text {. } \\
& \text { tbox:isInDomainOf(?ReqClass, ?pro }) \rightarrow \text { sqwrl:select(?pro). }
\end{aligned}
$$

In the above statements, RegClass is the design requirement class that tags instance $r_{\mathrm{i}}$, and pro denotes all of the properties that belong to RegClass. The variables and relationships in the above statements are illustrated in Fig. 9.

The retrieved design requirements above are only the ones proposed by an existing product in the knowledge base, but they can provide references for new product design. The knowledge framework also provides a user interface to append new design requirements or modify the retrieved design requirements.

\subsection{Knowledge retrieval for manufacturing processes}

As seen from Fig. 7, the manufacturing process instances of a product are linked by intermediate products, one after another, along the directionality of "inputs" and "outputs". As a result, a closed loop is formed from the selected material instance to the final product instance. It is evident that to retrieve all of the manufacturing process instances involved in the production of a product, it is necessary to execute an iterative query from the queried product instance. The iterative query steps are illustrated in Fig. 10. 
In Fig. 10, "MyProductClass" is the product class that the product to be designed belongs to, and all of the retrieved manufacturing process instances are stored in a two-dimensional array, QueryMfgProcess. By the manufacturing process instances, all of the information related to a manufacturing process can be obtained, including the constraints and the performance influence of the manufacturing process on the materials.

\section{A case study: constructing a mold material selection knowledge system}

To demonstrate the proposed methodology and framework, we take the construction of a mold material selection knowledge system as the case study. A mold is a type of tool used to shape objects; therefore, it is important technical equipment and has been widely used in industrial production. Due to the working condition and process requirements, the material selection has a significant influence on the quality, service life, and economy of the mold product. At the same time, it is well known that selecting a suitable mold material requires extensive material knowledge. The following will expatiate how this knowledge framework can help to construct a mold material selection knowledge system.

\subsection{Defining mold material selection concepts}

For the specific mold products, it is necessary to define some new concepts on mold material selection. In this knowledge framework, concepts are represented as 
ontology classes. For the sake of simplicity, the following will only discuss partial concept definitions on the hot working mold product, which includes the product concepts, design requirement concepts, and manufacturing process concepts.

First of all, we use a Molds class that is defined as a subclass of the EngProduct class to represent all of the concepts related to mold products. Then, according to their common characteristics, the Molds class derives its three subclasses, Hot-workingMolds class, Cold-workingMoldsclass and PlasticMolds class, as shown in Fig. 11 (a). In addition, the taxonomical semantics of the Molds class is defined as the knowledge rules, as shown in Table 3.

Furthermore, the analysis of the mold design requirements for materials is carried out, which mainly includes two aspects. One is to analyze the constraint requirements; as a result, we define a few mold design requirement classes as subclasses of the ConstraintRequirementclass. The other is to analyze the working failure of mold products. For hot working molds, as they usually work in high temperature and impact conditions, the failure mainly includes four types: cracking, wear, corrosion, and thermal fatigue. Preventing failure should be defined as the design objective for material selection. The definitions of mold design requirements for materials are shown in Fig. 11(b).

Manufacturing processes, costs, and environmental impacts also need to be analyzed, and some new concept classes are defined. All works on constructing new concepts are carried out in the knowledge constructor (Protégé editor) at the user interface layer. The fundamental ontology model for material selection is open and 
accessible. Hence, we can easily establish the concept model in this knowledge framework.

\subsection{Creating the knowledge instances for mold material selection}

In this knowledge framework, the instantiated facts of material selection knowledge are defined in the knowledge instance base (ABox). We can consult mold design experts, reference manuals, handbooks and other information sources to collect and capture the mold materialselection knowledge. Then, the captured knowledge is organized and taggedas knowledge instances by Protégé editor in human-computer interaction mode.

According to the concept model and the creating instance method introduced in Section 5, we collected more than 100 mold materials and related information on material properties, manufacturing processes, and product applications. The created mold material selection instances and the above concept definitions are stored in an OWL file (materials.owl). Fig. 12 illustrates partial instances of a material selection for a die-casting mold product.

\subsection{Knowledge-based mold material selection}

The knowledge-based mold material selection is carried out in the material selector at the user interface layer. The material selector is a prototype software tool that was developed based on Eclipse Java development tools and Protégé-OWL API and plays a communication role between design engineers and the knowledge reasoning layer. The interface of the material selector is as shown in Fig. 13, which 
includes three sub-windows: a product class window, a material selection window, and an output bar. The following will demonstrate how the material selector provides knowledge services for mold material selection.

\subsubsection{Starting the material selector}

To start the material selector, two program steps are needed. First, an SQWRL query engine must be created. In this step, the material selection knowledge base mentioned above is loaded, and an OWL model is created, which usually requires a uniform resource address for the knowledge base file. In this case, the knowledge base resource is at "http://www.jhcad.com/kbs/materials.owl". An SQWRL query engine is created by executing the constructor function of the SQWRLQueryEngineFactory class [23]. Then, all named mold product classes and their product instances in the knowledge base are listed in the product class tree window by their hierarchical relationships, as shown on the left side of the user interface.

\subsubsection{Retrieving a material selection}

In general, a material selection starts from the product to be designed. An existing material selection is usually considered a reliable reference for the material selection of similar products. As mentioned above, a material selection involves many aspects from a rough material to its manufacturing processes, the final finished product, and design requirements. The detailed knowledge retrieval methods have been introduced in Section 6. For example, in the case shown in Fig. 13, when 
"Copper_die_1" instance, which is an instance of the DieCastingMolds class, is selected, a material selection of the die-casting mold product is retrieved from the knowledge base. All of the instances associated with this material selection are listed in the corresponding list box in the material selector's window. We can continue to select the related instances to obtain the specific instance information. For example, when the "FormingObject_1" instance is selected, the attribute values related to its properties "formsMaterial" and "formsStructure" are retrieved and displayed in the edit box.

\subsubsection{Creating a new material selection}

On the basis of the above retrieval, according to the practical design requirements of the product to be designed, we can create a new material selection by modifying an existing material selection through the material selector. The information flow of creating a new material selection is illustrated in Fig. 14, and the creating steps are as follows:

Step1. Select a product class or an instance from the product class hierarchy shown in the product view window.

The design requirements proposed by the selected product class are automatically retrieved and chosen as the new design requirement reference, some of which can be modified, deleted, or appended. In this case, we select a suitable material for an aluminum alloy die-casting mold product. The design requirements and manufacturing processes are retrieved from the knowledge base and listed in the user 
interface. Then, we can modify the forming material from "CuZn35" to "6061", the production quantity from " 10000 " to " 5000 ", and so on.

Step2. Obtain the product's property requirements for materials.

In this step, the new design requirements and manufacturing requirements can be automatically mapped to the material property requirements. The mapping procedures are as follows:

(1) Defining the mapping knowledge rules.

First, we employ SWRL to encode the mapping rules. The antecedent part describes the design requirements, and the consequent part describes the material property requirements. Table 4 gives the partial mapping rules.

(2) Creating the requirement instances of the design and manufacturing processes.

As the construction of new design and manufacturing requirements is carried out on the guidance of this ontology model, each requirement can be easily found in its related ontology class. Hence, we use the Jena API [41] to create the design requirement instances, according to newly modified design requirements. For example, in this case, we need to respectively create instances of the FormingObject class, ProductionQuanitity class, WorkTemperature class, and so on.

(3) Creating the material property instances with mapping knowledge rules.

To realize the mapping from design requirements to material property requirements, we need to perform knowledge reasoning for the design requirement instances created above. The inference mechanism of SWRL is to check each atom 
in the rule whether it is satisfied by an interpretation under the conditions given in the Interpretation Conditions Table [36]. In fact, in the SWRL rules shown in Table 4, only the antecedent part of each rule may hold. As there is no property instance that has been created so far, it is impossible to reason the consequent part. Hence, we only reason the antecedent part of each rule with the design requirement instances created above. If the matching reasoning succeeds, each concept atom in the consequent part of the rule will be defined as an instance of the material property class. For example, if the antecedent part of Rule 1 in Table 4 holds, some instances of the material's thermal properties, such as the hot hardness instance and the hot yield strength instance, will be created.

Step3. Retrieve materials that can meet the proposed property requirements from the existing material selections.

From the above steps, we have obtained the material property requirements proposed by the product to be designed in the form of instances. As there are comparison operations between material property values, we first define two concepts with SWRL rules to represent retrieving of two types of comparison operation. Then, we construct two selection sets by executing two types of comparison operation. Finally, we calculate the intersection set of the two selection sets. The intersection set is the final result of retrieving the materials. Here, we use a built-in called sqwrl:makeSet to construct a selection set. The SWRL rules and SQWRL querying statements are as follows: 
- Rule 1: This rule is used to select materials whose property values are greater than or equal to the values required by design requirements.

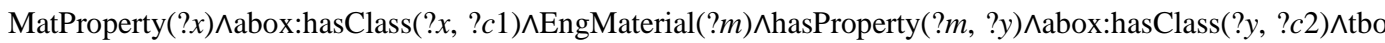

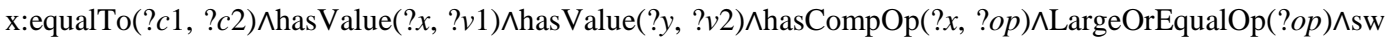
rlb:greaterThanOrEqual $(? v 2, ? v 1) \rightarrow$ largeOrEqualCompare $(? x, ? m)$

- Rule 2: This rule is used to select materials whose property values are less than the values required by design requirements.

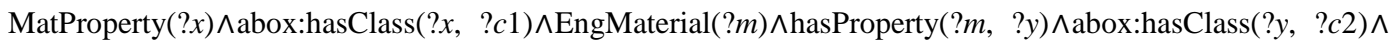

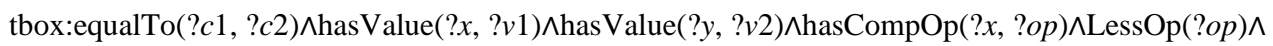
swrlb:lessThan $(? v 2, ? v 1) \rightarrow$ lessCompare $(? x, ? m)$

- Querying statement: This statement is to construct the retrieved set from two types of comparison operation and to calculate their intersection set.

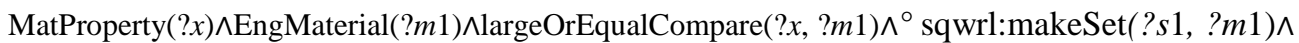

EngMaterial(?m2)^lessCompare $(? x, ? m 2) \wedge^{\circ} \operatorname{sqwrl}: m a k e S e t(? s 2, ? m 2) \wedge$

sqwrl:intersection $(? s 3, ? s 1, ? s 2) \wedge$ EngMaterial $(? m) \wedge$ swrlb:equal $(? m, ? s 3) \rightarrow \operatorname{sqwrl}: \operatorname{select}(? m)$

In the above query statement, $x$ denotes the property requirements for materials, $s 3$ denotes an intersection of two sets obtained from two types of comparison operation, and $m$ is a list of the retrieved materials.

Step4 (optional step). Query an external material database.

To select many more materials, the material selector can be asked to query an external material database. Database technologies have many advantages in managing data information. In this case, we have set up a mold material database with Microsoft Access 2010, in which the database fields are designed in terms of mold material property requirements. Currently, the most commonly used approach to query a relational database is to use Structured Query Language (SQL) query, 
which requires sending an SQL statement to the material database system. An SQL query has a fixed format. A "Select" query can be represented in the following form:

SELECT material_name FROM database_name WHERE conditional_clause.

The "conditional_clause" is a statement that includes a comparison predicate, which restricts the rows returned by the query. In fact, the conditional clause can be represented as a conjunctive combination by a set of material property constraints. Hence, it is easy to automatically construct the conditional clause according to the property requirements of the product to be designed. The results returned are considered the candidate materials and added to the candidate material set.

Step5. Rank candidate materials.

For all candidate materials in the material selection set, it is necessary to implement a ranking operation. In general, according to the objective design requirements, a multiple attribute decision-making can be carried out. In this material selector, we employ a commonly used approach, the analytic hierarchy process (AHP) approach, to rank the candidate materials [3]. At the same time, the material selector can provide a multiple objective design attribute data file in XML format to integrate other multiple attribute decision-making approaches.

Step6. Create a new material selection.

After selecting a new desired material, we can use the Jena API [41] to create all instances of the ontology concepts associated with the selected material. The newly created material selection can be appended to the knowledge base for future reuse. 
It needs to be noted that the whole process of creating a new material selection is carried out in the material selector of this knowledge framework, and the user only needs to define the mapping knowledge rules.

\subsection{Discussion}

The case study shows that the knowledge framework presented in this paper can provide the following benefits for constructing a domain-oriented material selection knowledge system:

(1) A semantic representation of the material selection knowledge.

First, this framework provides an ontology-based concept model that can semantically represent the material selection knowledge spread across the domains of the material, design, and manufacturing as well as product applications at the conceptual level. Second, the specific material selection knowledge is represented as a set of labeled instances and RDF instance graphs in terms of the concept model, which provides a formal approach to organizing the captured material selection knowledge. Moreover, the material selection knowledge with causal relations is defined as knowledge rules encoded with SWRL, which enrich the knowledge representation for material selection. This knowledge framework can effectively combine the above knowledge concepts, instances, and rules.

The constructed knowledge base is stored in a shared network namespace so that users from different applications can inherit, create, and modify the knowledge base in terms of the material selection knowledge of their domain. 
(2) Knowledge-retrieving services for material selection.

Based on the constructed knowledge base, this framework provides knowledge-retrieving services for material selection. The knowledge-retrieving services include retrieving design requirements and manufacturing processes, knowledge-mapping from design requirements to material properties, and screening materials. The framework program to realize knowledge-retrieving services is independent of the specific material selection applications.

(3) Improving the scalability of the knowledge-based material selection system.

As a wide variety of engineering materials requires much application domain knowledge, it is very important for a knowledge system to have good scalability. This work improves the scalability of the knowledge system from the following aspects:

- The representation model provides a concept framework based on an ontology.

Based on the concept framework, users can easily define new subclasses and properties in the base class, according to their domain knowledge requirements. For example, as introduced in the case study, the new Mold class can be defined as a subclass of the EngProduct class. Meanwhile, a set of knowledge instances for mold material selection are easily defined in the ABox of the knowledge base.

- New material concepts and mapping relations can be explicitly defined by SWRL rules.

Many new concepts and mapping knowledge resulting from causal relations can be defined by SWRL rules, which greatly extend the knowledge representation 
capability. For example, as mentioned in Section 5, new concepts, such as lightweight material, wear-resistant material and various processing schemes, can be easily defined and combined in the original concept framework.

- No need to modify the application program of the knowledge system.

Compared with traditional knowledge representation methods, modifying or extending the material selection knowledge is only carried out in the knowledge editor and does not need to modify any framework program. As mentioned in the above case study, the program to retrieve materials is independent of the specific application. The query operation only depends on the external knowledge base.

In addition, the presented knowledge representation is based on description logic. Description logic is a mathematically rigorous representation that enables reasoning to be performed on the ontology including concept consistency, concept equivalence, and concept subsumption.

\section{Conclusion}

The selection and application of engineering materials requires much material selection knowledge. In the face of the vast number of materials, it is very necessary to research and develop an open, formal, and shared knowledge representation and knowledge framework for material selection. The ontology-based Semantic Web technologies have many advantages over other concept modeling methods in classification, sharing, and formalization, which provide a promising solution to addressing current challenges. 
In this paper, the basic concepts and relationships involved in all aspects of engineering material selection are analyzed in detail. The Semantic Web technology is introduced into the knowledge representation for material selection, and a novel ontology-based knowledge framework is proposed. The knowledge framework provides a novel ontology-based concept model for representing the material selection knowledge. Under this knowledge framework, it is easy to build up the material selection knowledge base for different application domains. Furthermore, the knowledge framework can provide powerful knowledge services for material selection. The case study demonstrates that the presented knowledge framework can help users effectively construct a domain-oriented material selection knowledge system.

The completed works in this paper lay a foundation for constructing an open and shared engineering material knowledge framework. In future works, research on the Web-based capturing and modeling of material selection knowledge will be carried out. In addition, the concept model for material selection should consider more aspects, such as the final stages of a product's existence (e.g., remanufacturing or recycling).

\section{Acknowledgement}

This work is supported by the National Science Foundation of China (Grant No. 60773214 and Grant No. 51375069). The authors thank the anonymous reviewers for their helpful suggestions on this study. 


\section{References}

[1] M. Ashby, H. Shercliff, D. Cebon, Materials engineering, science, processing and design. Oxford: Elsevier, 2007.

[2] J.C. Albinana, C. Vila, A framework for concurrent material and process selection during conceptual product design stages, Mater. Des.41(2012) 433-446.

[3] A. Jahan , M.Y. Ismail, S.M. Sapuan, F. Mustapha, Material screening and choosing methods - A review, Mater. Des. 31( 2010) 696-705.

[4] P.S. Ramalhete, A.M.R. Senos, C. Aguiar, Digital tools for material selection in product design, Mater. Des.31(2010) 2275-2287.

[5] W.J.C. Verhagen, P. Bermell-Garcia, R.E.C.V. Dijk, R., Curran. A critical review of knowledge-based engineering: An identification of research challenges, Adv. Eng. Inform. 26 (2012) 5-15.

[6] P. Witherell, S. Krishnamurty, I.R. Grosse, J.C. Wileden, Improved knowledge management through first-order logic in engineering design ontologies, Artif. Intell.Eng. Des.Anal. Manuf. 24(2010) 245-257.

[7] F. Ameri, B. Kulvatunyou, N. Ivezic, K. Kaikhah, Ontological conceptualization based on the SKOS, J. Comput. Inf. Sc. Eng., 14(3) (2014) 031006 (11 pages)

[8] S. Ma, L. Tian, Ontology-based semantic retrieval for mechanical design knowledge, Int. J. Comput. Integr. Manuf. 28(2) (2015) 226-238.

[9] C. Bock, X. Zha, H.-W.Suh, J.-H.Lee, Ontological product modeling for collaborative design, Adv. Eng. Inform.24 (4) (2010) 510-524. 
[10] V. Goel, J.H. Chen, Application of expert network for material selection in engineering design, Comput. Ind. 30(2) (1996) 87-101.

[11] S. M. Sapuan, H.S.Abdalla, A prototype knowledge-based system for the material selection of polymeric-based composites for automotive components, Compos Part A. 29A(1998) 731-42.

[12] M. L. Sanyang, S. M. Sapuan, Development of expert system for biobased polymer material selection: food packaging application, J Food Sci Technol. DOI: $10.1007 / \mathrm{s} 13197-015-1759-6(2015)$

[13] M.H.F. Zarandi, S. Mansour, S.A. Hosseinijou, M. Avazbeigi, A material selection methodology and expert system for sustainable product design, Int. J. Adv. Manuf. Technol. 57(2011) 885-903.

[14] M. Ipek, I.H. Selvi, F. Findik, O. Torkul, I.H. Cedimoglu, An expert system based material selection approach to manufacturing, Mater. Des.47(2013) 331-340.

[15] C. Urrea, G. Henriquez, M. Jamett, Development of an expert system to select materials for the main structure of a transfer crane designed for disabled people, Expert. Syst. Appl. 42(1) (2015) 691-697.

[16] X.F. Zha, A web-based advisory system for process and material selection in concurrent product design for a manufacturing environment, Int J AdvManuf Technol. 25(3-4)(2005) 233-243.

[17] S. Kumar, R. Singh, A short note on an intelligent system for selection of materials for progressive die components, J Mater Process Technol. 
182(1-3)(2007) 456-461.

[18] R. Amen, P. Vomacka, Case-based reasoning as a tool for materials selection, Mater Des.22(2001)353-358.

[19] A.M.M. Ullah, K.H. Harib, An intelligent method for selecting optimal materials and its application, Adv. Eng. Inform. 22 (2008) 473-483.

[20] T. Gruber, A translation approach to portable ontology specifications, Knowledge Acquisition. 5(2)(1993) 199-220.

[21] W.N. Borst, Construction of engineering ontologies for knowledge sharing and reuse, Ph.D. thesis, University of Twente, Netherlands, 1997.

[22] P. Zhan, U. Jayaram, O. Kim, L. Zhu, Knowledge representation and ontology mapping methods for product data in engineering applications, ASME J. Comput. Inf. Sci. Eng. 10(2010) 1-11

[23] Y. Zhang, X. Luo, H. Zhang, J.W. Sutherland, A knowledge representation for unit manufacturing processes, Int. J. Adv. Manuf. Technol. 73(2014)1011-1031.

[24] A. Matsokis , D. Kiritsis, An ontology-based approach for Product Lifecycle Management, Comput. Ind. 61(8) (2010) 787-797.

[25] I.O. Sanya, E.M. Shehab, An ontology framework for developing platform-independent knowledge-based engineering systems in the aerospace industry, Int. J. Prod. Res. 52(2014) 6192-6215.

[26] A. Varde, E. Begley, S. Fahrenholz-Mann, MatML: XML for information exchange with materials property data, in: Proceedings of the 4th International Workshop on Data Mining Standards, Services and 
Platforms, 2006, pp. 47-54.

[27] T. Ashino, Materials ontology: An infrastructure for exchange materials information and knowledge, Data Sci. J. 9(2010) 54-61.

[28] T. Rahmani, S. Bougain, D. Gerhard, Ontology-based integration of heterogeneous material data resources in product lifecycle management, in: Proceedings of the 2013 IEEE International Conference on Systems, Man, and Cybernetics, Vienna, Austria, 2013.

[29] X. Cheng, C. Hu, Yang Li, Semantic-driven knowledge representation model for the materials engineering application, Data Sci. J. 13(2014) 26-44.

[30] T. Ashino, M. Fujita, Definition of a Web ontology for design-oriented material selection, Data Sci. J. 5(2006) 52-63.

[31] M. Liu, W. Shen, Q. Hao, J. Yan, L. Bai, A fuzzy matchmaking approach for Semantic Web Services with application to collaborative material selection, Comput. Ind. 63(2012)193-209.

[32] V. Premkumar, S. Krishnamurty, J.C. Wileden, I.R. Grosse, A semantic knowledge management system for laminated composites, Adv. Eng. Inform.28 (2014) 91-101.

[33] R.J. Brachman, H.J. Levesque. Knowledge representation and reasoning. San Francisco: Elsevier/ Morgan Kaufmann, 2004.

[34] P.M. Sargent, Engineering material information system, Eng. Comput. 8(1992) 243-252.

[35] M.K. Smith, C. Welty, D.L. McGuinness, OWL Web ontology language guide, 
2004. <http://www.w3.org/TR/owl-guide/> (retrieved 08.02.14)

[36] I. Horrocks, P.F. Patel-Schneider, H. Boley, S. Tabet, B. Grosof, M. Dean, SWRL: A semantic web rule language combining OWL and RuleML. 2004. <http://www.w3.org/Submission/SWRL> (retrieved 12.05.04)

[37] M.J. O'Connor, A.K. Das, SQWRL: a query language for OWL. In: OWL: experiences and directions (OWLED), Fifth International Workshop, Chantilly, VA, 2009.

[38] L. Zhu, U. Jayaram, O. Kim, Semantic applications enabling reasoning in product assembly ontologies—moving past modeling, Comput. Inf. Sc. Eng., 12(3) (2012) 011009 (17 pages)

[39] A. Garcia-Crespo, B. Ruiz-Mezcua, J.L. Lopez-Cuadrado, J.M. Gomez-Berbis, Conceptual model for semantic representation of industrial manufacturing processes. Comput. Ind. 61 (2010) 595-612.

[40] X. Fiorentini, R. Sudarsan, H. Suh, J. Lee, R.D. Sriram, An analysis of description logic augmented with domain rules for the development of product models, Comput. Inf. Sc. Eng., 10 (2010) 021008 (13 pages).

[41] M. Horridge, S. Bechhofer, The OWL API: A Java API for OWL ontologies, Semantic Web, 2(2011) 11-21 


\section{Table 1}

Partial knowledge rules for defining material concepts

\begin{tabular}{|c|c|c|c|}
\hline No. & Material concepts & SWRL rules & Explanation \\
\hline \multirow[t]{4}{*}{1} & LightweightMaterial & $\operatorname{EngMaterial}(? x) \wedge$ hasProperty $(? x, ? p) \wedge \operatorname{Density}(? p)$ & A material with a density \\
\hline & & ^hasValue $(? p, ? y) \wedge$ swrlb:lessThanOrEqual(?y, & less than $2.7 \mathrm{~g} / \mathrm{cc}$ is \\
\hline & & 2.7) $\wedge$ hasUnit $(? y, ? u) \wedge$ UnitG/cc $(? u) \rightarrow$ & defined as a lightweight \\
\hline & & LightweightMaterial (?x) & material. \\
\hline \multirow[t]{5}{*}{2} & ThermalInsulationMaterial & EngMaterial $(? x) \wedge$ hasProperty $(? x, ? p) \wedge$ & A material with a thermal \\
\hline & & ThermalConductivity $(? p) \wedge$ hasValue $(? p, ? v) \wedge$ & conductivity less than \\
\hline & & swrlb:lessThan $(? v, 0.05) \wedge$ hasUnit $(? p, ? u) \wedge$ & $0.05 \mathrm{~W} / \mathrm{m}-\mathrm{K}$ is defined as \\
\hline & & UnitW/mk $(? u) \rightarrow$ ThermalInsulationMaterial(?x) & a thermal insulation \\
\hline & & & material. \\
\hline
\end{tabular}

\section{Table 2}

Partial knowledge rules for defining manufacturing capability concepts

\begin{tabular}{|c|c|c|c|}
\hline No. & Material concepts & SWRL rules & Explanation \\
\hline 1 & MachinableMaterial & $\begin{array}{l}\text { EngMaterial }(? x) \wedge \operatorname{Metal}(? x) \wedge \text { hasProperty }(? x, ? p) \wedge \\
\text { Hardness }(? p) \wedge \text { hasValue }(? p, ? y) \wedge \text { swrlb:lessThanOrEqual } \\
(? y, 80) \wedge \text { hasUnit }(? y, ? u) \wedge \text { UnitHRC }(? u) \rightarrow \\
\text { MachinableMaterial }(? x)\end{array}$ & $\begin{array}{l}\text { A metal material with hardness less } \\
\text { than } 80 \mathrm{HRC} \text { is defined as a } \\
\text { machinable material. }\end{array}$ \\
\hline 2 & CastingMaterial & $\begin{array}{l}\text { EngMaterial }(? x) \wedge \text { Metal }(? x) \rightarrow \text { CastinMaterial }(? x) \\
\text { EngMaterial }(? x) \wedge \text { Polymer }(? x) \rightarrow \text { CastinMaterial }(? x)\end{array}$ & $\begin{array}{l}\text { Metal materials and polymeric } \\
\text { materials are defined as casting } \\
\text { materials. }\end{array}$ \\
\hline 3 & WeldableMaterial & $\begin{array}{l}\text { EngMaterial }(? x) \wedge \operatorname{Metal}(? x) \wedge \text { hasComposition }(? x, ? e) \wedge \\
\text { Carbon }(? e) \wedge \text { hasPercent }(? e, ? v) \wedge \\
\text { swrlb:lessThan }(? v, 0.3) \rightarrow \text { WeldableMaterial }(? x)\end{array}$ & $\begin{array}{l}\text { A metal material with less than } \\
0.3 \% \text { carbon is defined as a } \\
\text { weldable material. }\end{array}$ \\
\hline
\end{tabular}




\section{Table 3}

Partial taxonomic semantic definitions for mold product concepts

\begin{tabular}{|c|c|c|c|}
\hline No. & Mold Concepts & SWRL Rules & Explanation \\
\hline 1 & Hot-workingMolds & $\begin{array}{l}\text { Molds }(? x) \wedge \operatorname{proposes}(? x, ? r 1) \wedge \text { FormingObject }(? r 1) \wedge \\
\text { formsMaterial }(? r 1, ? m) \wedge \operatorname{Metal}(? m) \wedge \operatorname{propose}(? x, r 2) \wedge \\
\text { WorkTemperature }(? r 2) \wedge \text { hasValue }(? r 2, ? z) \wedge \\
\text { swrlb:greaterThan }(? z, 300) \wedge \text { hasUnit }(? y, ? u) \wedge \\
\text { UnitCentigrade }(? u) \rightarrow \text { Hot-workingMolds }(? x)\end{array}$ & $\begin{array}{l}\text { Molds working at } \\
\text { temperatures higher than } \\
300 \text { degrees centigrade are } \\
\text { defined as hot-working } \\
\text { molds. }\end{array}$ \\
\hline 2 & Cold-workingMolds & $\begin{array}{l}\text { Molds }(? x) \wedge \text { moldMat }(? x, ? m) \wedge \operatorname{Metal}(? m) \wedge \text { workIn }(? x, \\
? y) \wedge \text { WorkTemperature }(? y) \wedge \text { hasValue }(? y, ? z) \wedge \\
\text { swrlb:lessThanOrEqual }(? z, 300) \wedge \operatorname{hasUnit}(? y, ? u) \wedge \\
\text { UnitCentigrade }(? u) \rightarrow \text { Cold-workingMolds }(? x)\end{array}$ & $\begin{array}{l}\text { Molds working at } \\
\text { temperatures less than or } \\
\text { equal to } 300 \text { degrees } \\
\text { centigrade are defined as } \\
\text { cold-working molds. }\end{array}$ \\
\hline 3 & PlasticMolds & $\begin{array}{l}\text { Molds }(? x) \wedge \operatorname{moldMat}(? x, ? m) \wedge \text { Polymer }(? m) \\
\rightarrow \text { PlasticMolds }(? x)\end{array}$ & $\begin{array}{l}\text { Molds injected with } \\
\text { polymer materials are } \\
\text { defined as plastic molds. }\end{array}$ \\
\hline
\end{tabular}

\section{Table 4}

\section{Partial knowledge rules for mapping design requirements}

\begin{tabular}{|c|c|}
\hline No. & SWRL Rules \\
\hline \multirow[t]{4}{*}{1} & $\begin{array}{l}\text { FormingObject }(? r 1) \wedge \text { formsMaterial }(? r 1, ? m) \wedge \text { CuAlloy }(? m) \wedge \text { WorkTemperature }(? r 2) \wedge \text { hasValue }(? r 2, ? z 1) \wedge \\
\text { swrlb:greaterThan }(? z 1,550) \wedge \text { ProductionQuantity }(? r 3) \wedge \text { swrlb:greaterThan }(? r 3,5000)\end{array}$ \\
\hline & $\rightarrow$ HotHardness $(? p 1) \wedge$ hasValue $(? p 1,35) \wedge$ hasCompOp $(? p 1, ? o p 1) \wedge$ LargeOrEqualOp(?op1) $\wedge$ HotYield(?p2) \\
\hline & $\wedge$ hasValue $(? p 2,420) \wedge$ hasCompOp $(? p 2, ? o p 2) \wedge$ LargeOrEqualOp(?op2) $\wedge$ AntiMeltingLoss $(? p 3) \wedge$ \\
\hline & hasValue $(? p 3,35) \wedge$ hasCompOp(?p3, ?op3) ^LessOp(?op3) \\
\hline \multirow[t]{5}{*}{2} & FormingObject $(? r l) \wedge$ formsMaterial $(? r l, ? m) \wedge$ AlAlloy $(? m) \wedge$ WorkTemperature $(? r 2) \wedge$ has Value $(? r 2, ? z l) \wedge$ \\
\hline & swrlb:greaterThan $(? z 1,500) \wedge$ ProductionQuantity $(? r 3) \wedge$ swrlb:greaterThan $(? r 3,10000)$ \\
\hline & $\rightarrow$ HotHardness $(? p 1) \wedge$ hasValue $(? p 1,30) \wedge$ hasCompOp $(? p 1, ? o p 1) \wedge$ LargeOrEqualOp $(? o p 1) \wedge$ HotYield(?p2) \\
\hline & $\wedge$ hasValue $(? p 2,400) \wedge$ hasCompOp $(? p 2, ? o p 2) \wedge$ LargeOrEqualOp(?op2) $\wedge$ AntiMeltingLoss $(? p 3) \wedge$ \\
\hline & hasValue $(? p 3,30) \wedge$ hasCompOp(?p3, ?op3) ^LessOp(?op3) \\
\hline
\end{tabular}






Fig. 1. An ontology-based knowledge framework for material selection

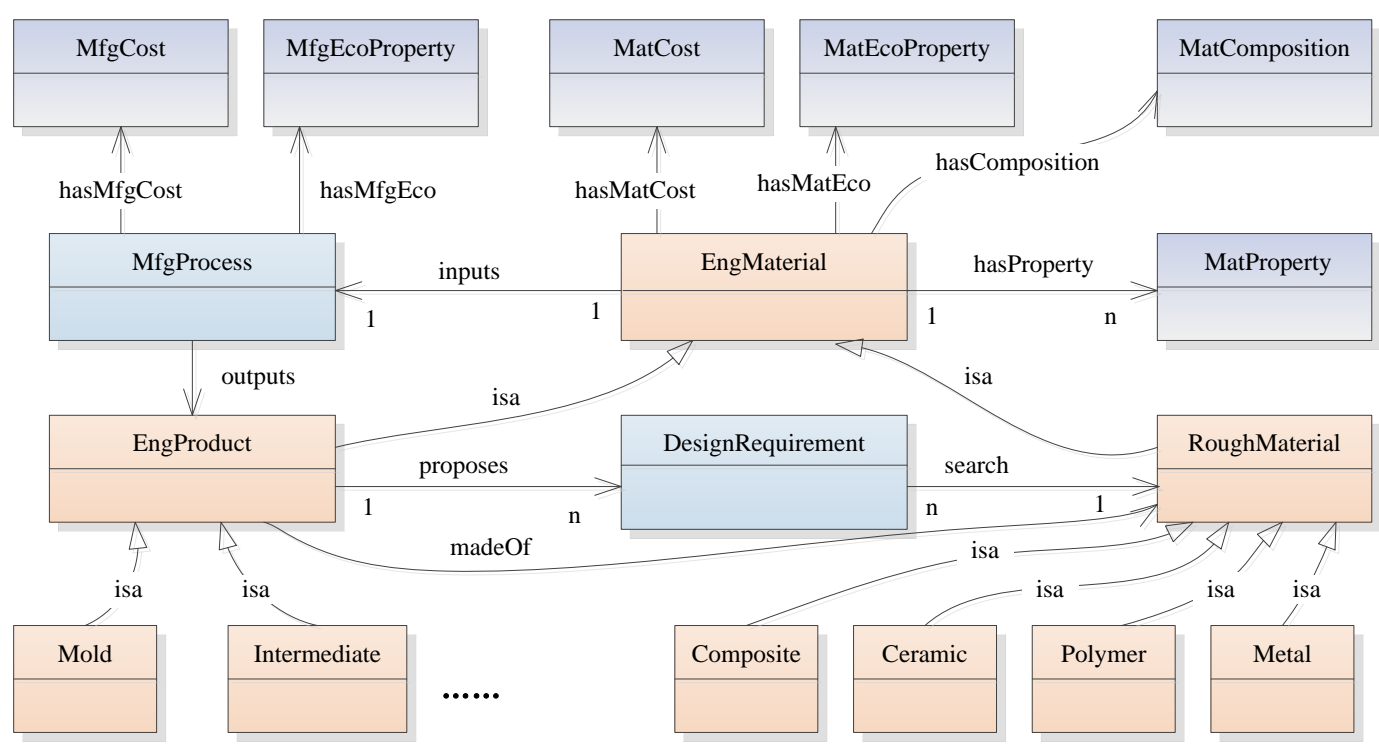

Fig. 2.An ontology-based concept model for material selection 


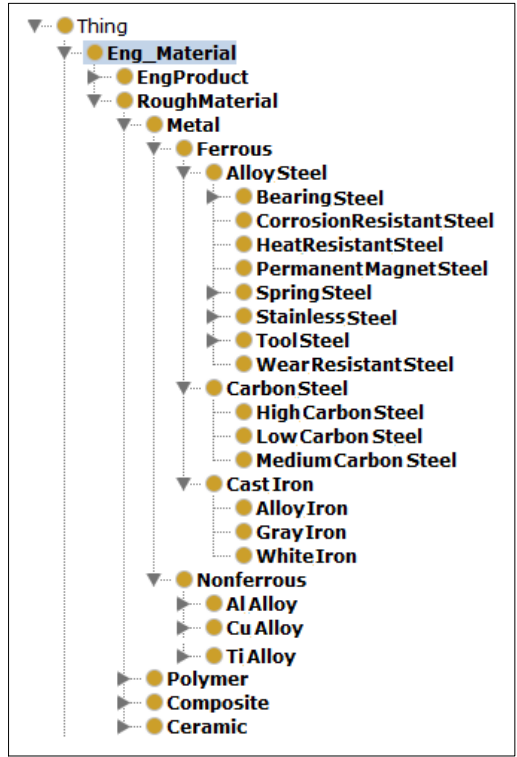

(a)

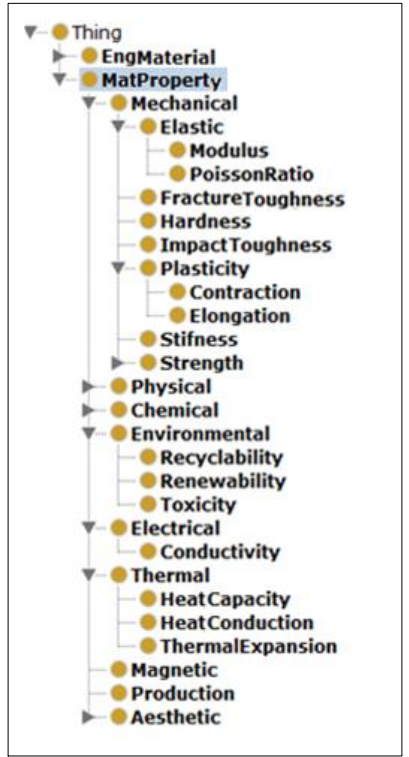

(b)

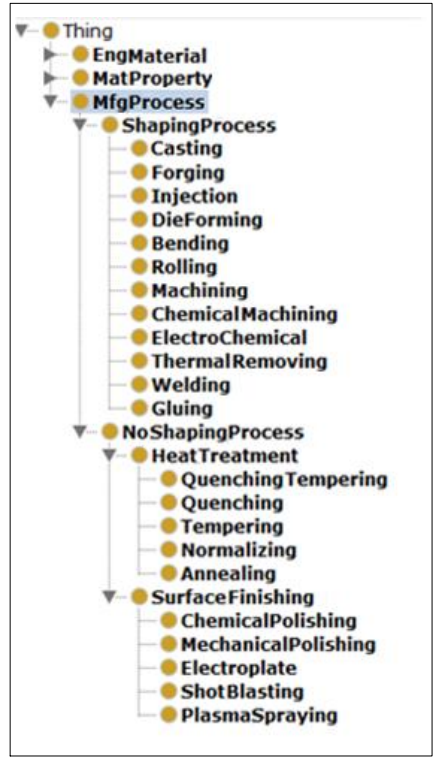

(c)

Fig. 3. The taxonomy of materials, material properties, and manufacturing processes

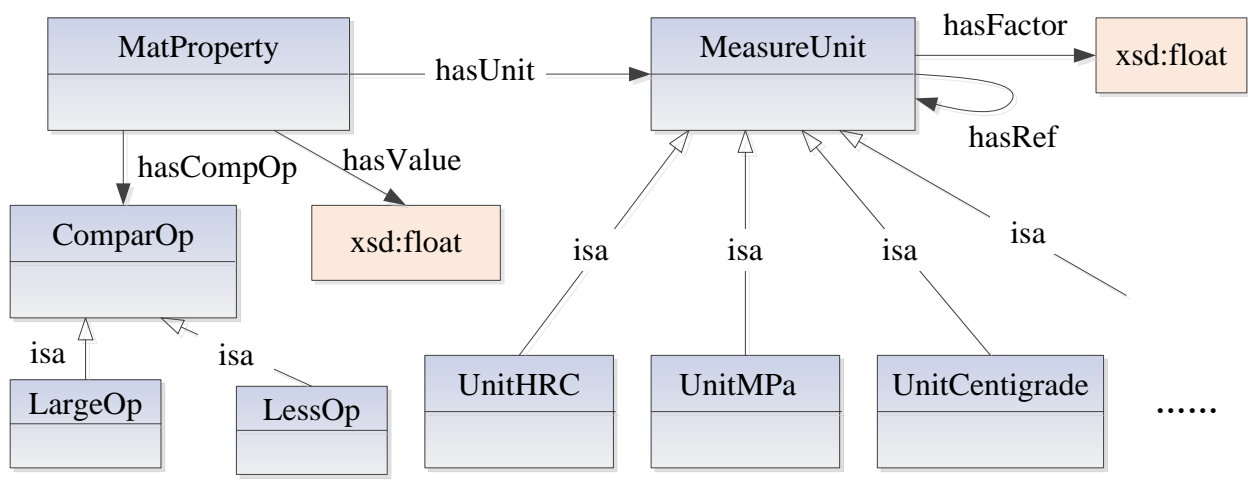

Fig. 4. The definition of the MatProperty class

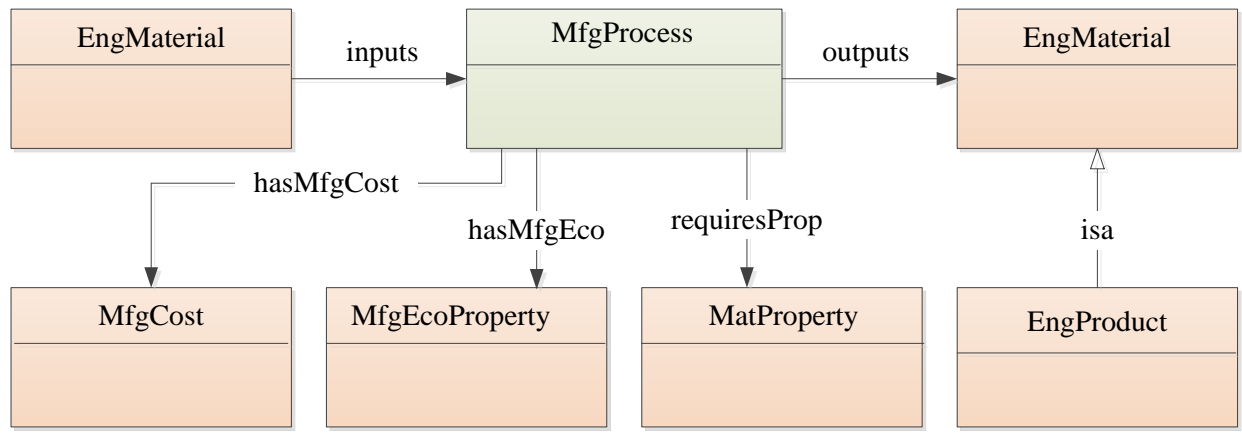

Fig. 5.Relationships between manufacturing processes and materials 


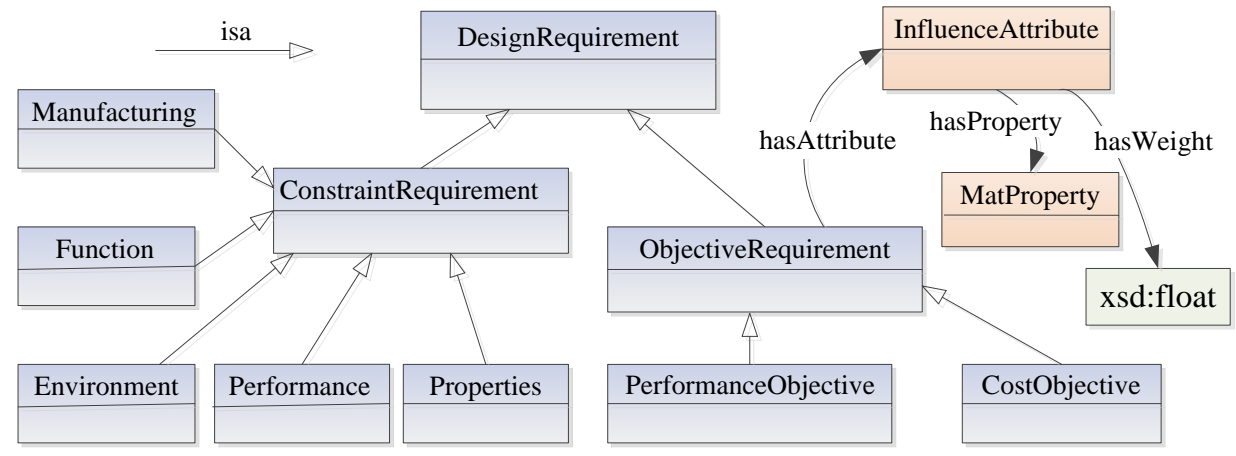

Fig. 6.Definition of theDesignRequirement class

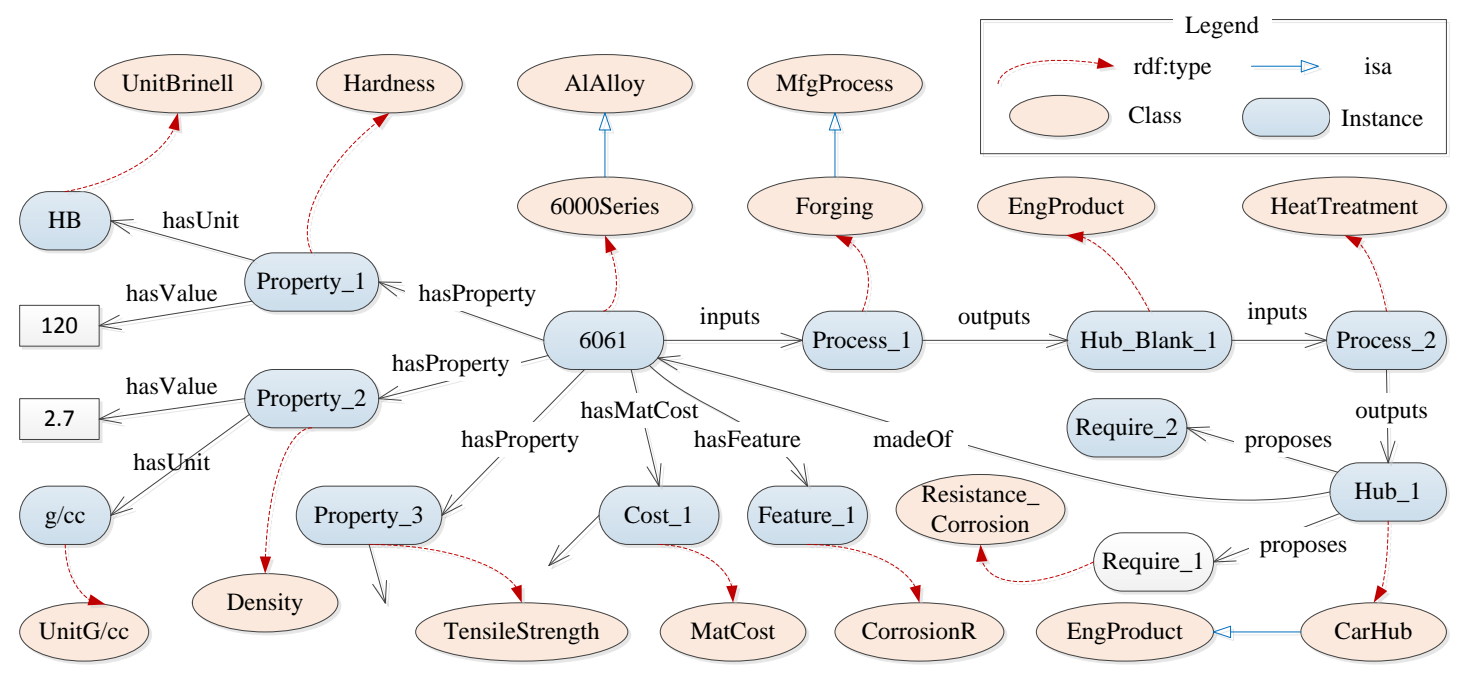

Fig.7.An RDF graph for the material selection of a car hub product

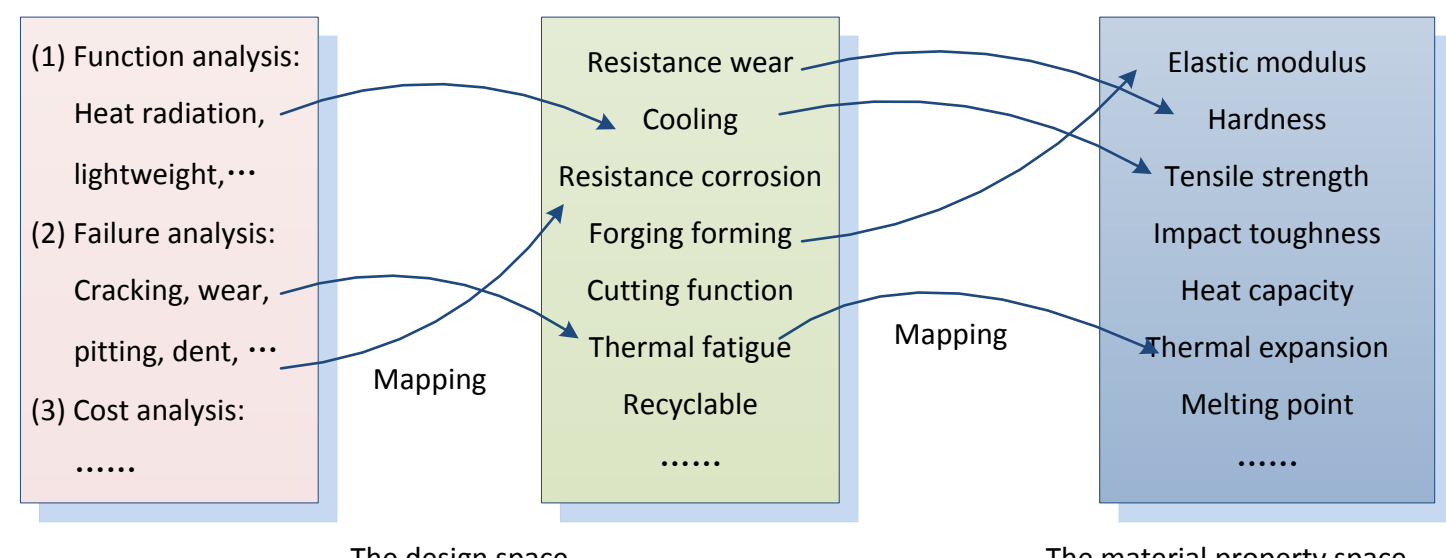

The design space

The material property space

Fig.8.Mapping relations between the design space and the property space 


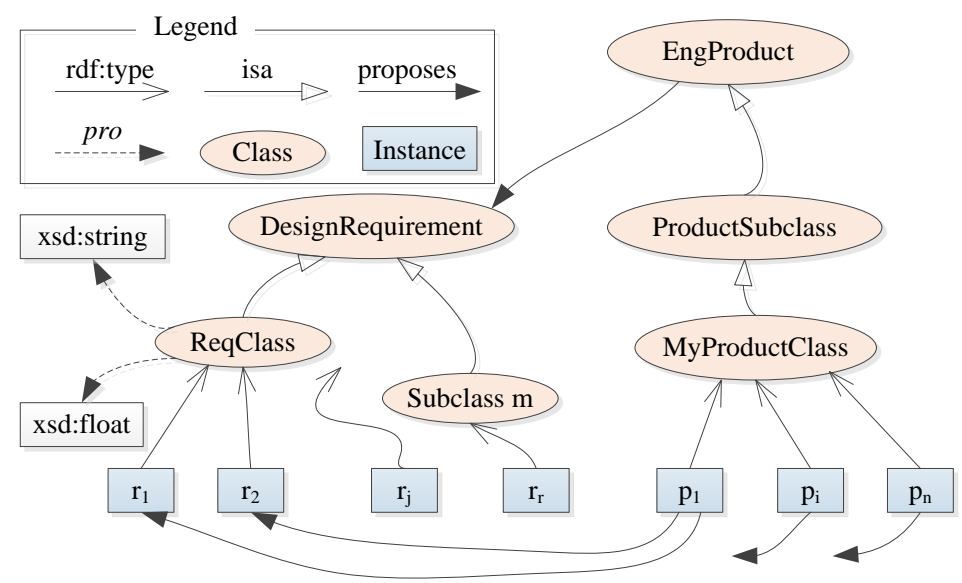

Fig. 9.Semantic relationships between the selected product and its design requirements

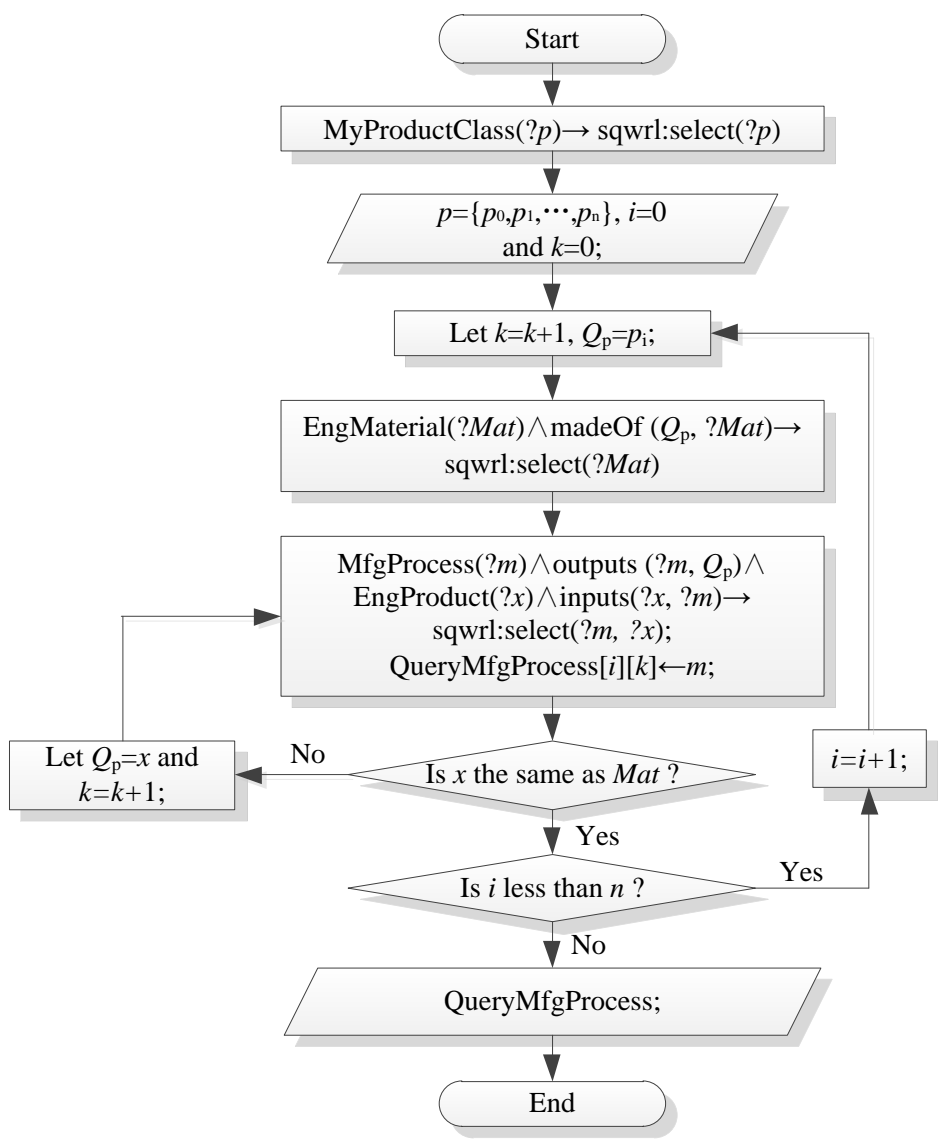

Fig. 10.A flow diagram for retrieving the manufacturing process instances 


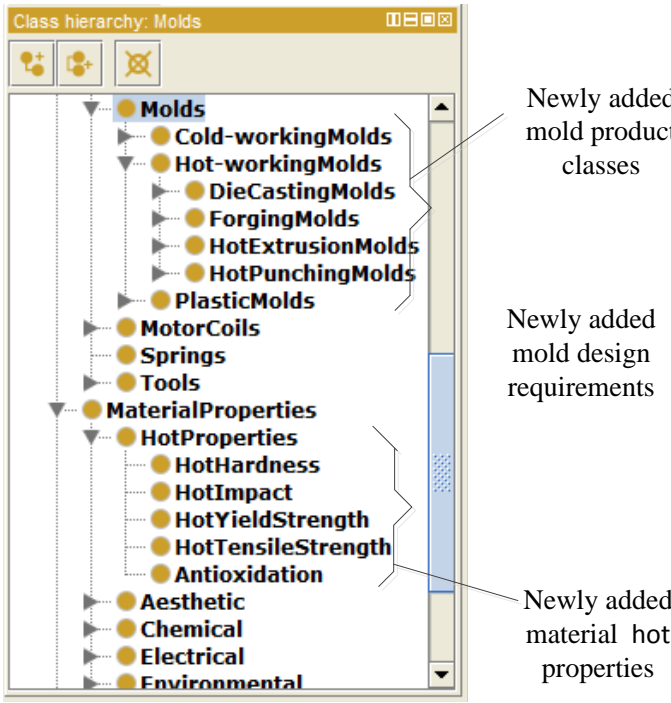

(a)

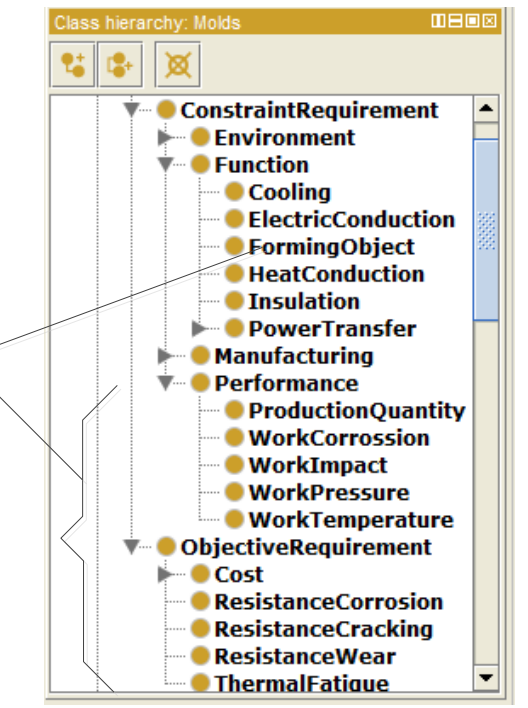

(b)

Fig. 11.Newly defined mold product classes and design requirement classes

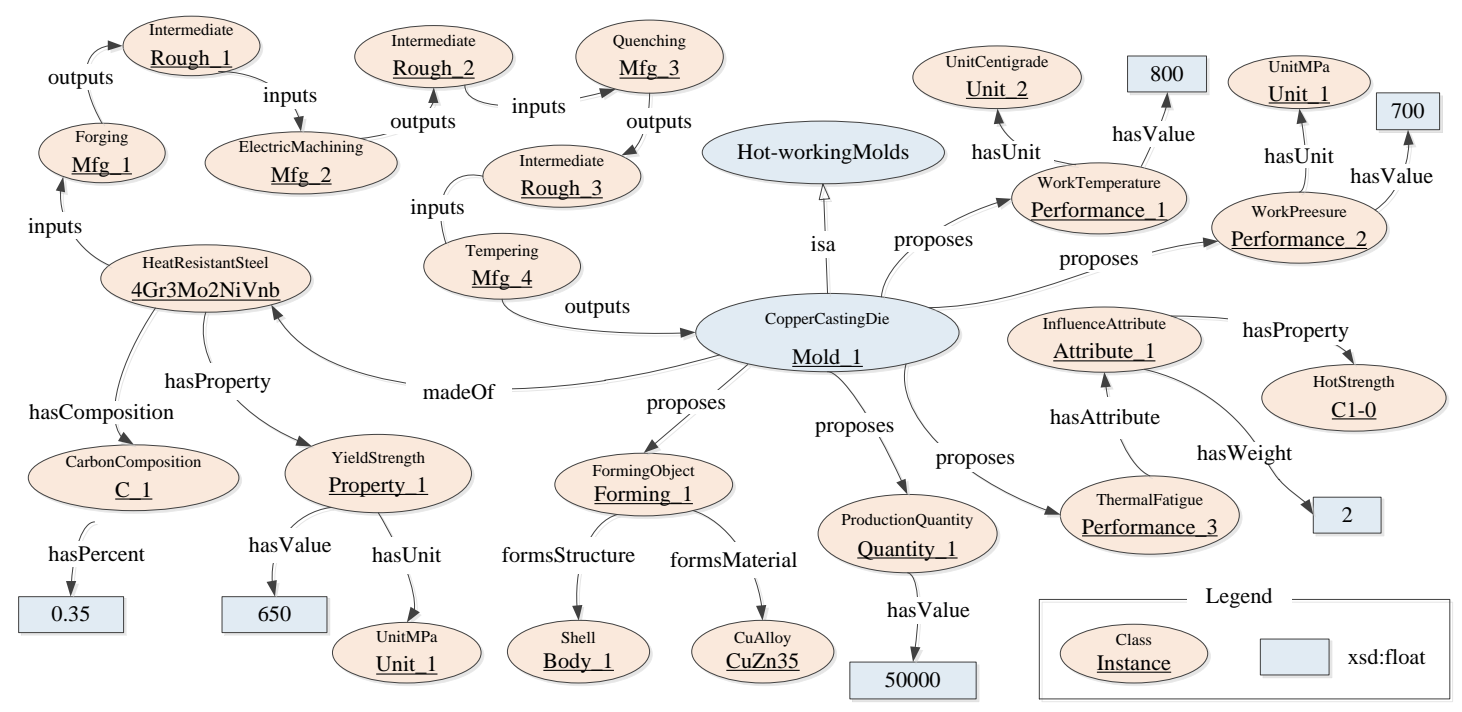

Fig. 12.Partial instances of a material selection fora die-casting mold product 


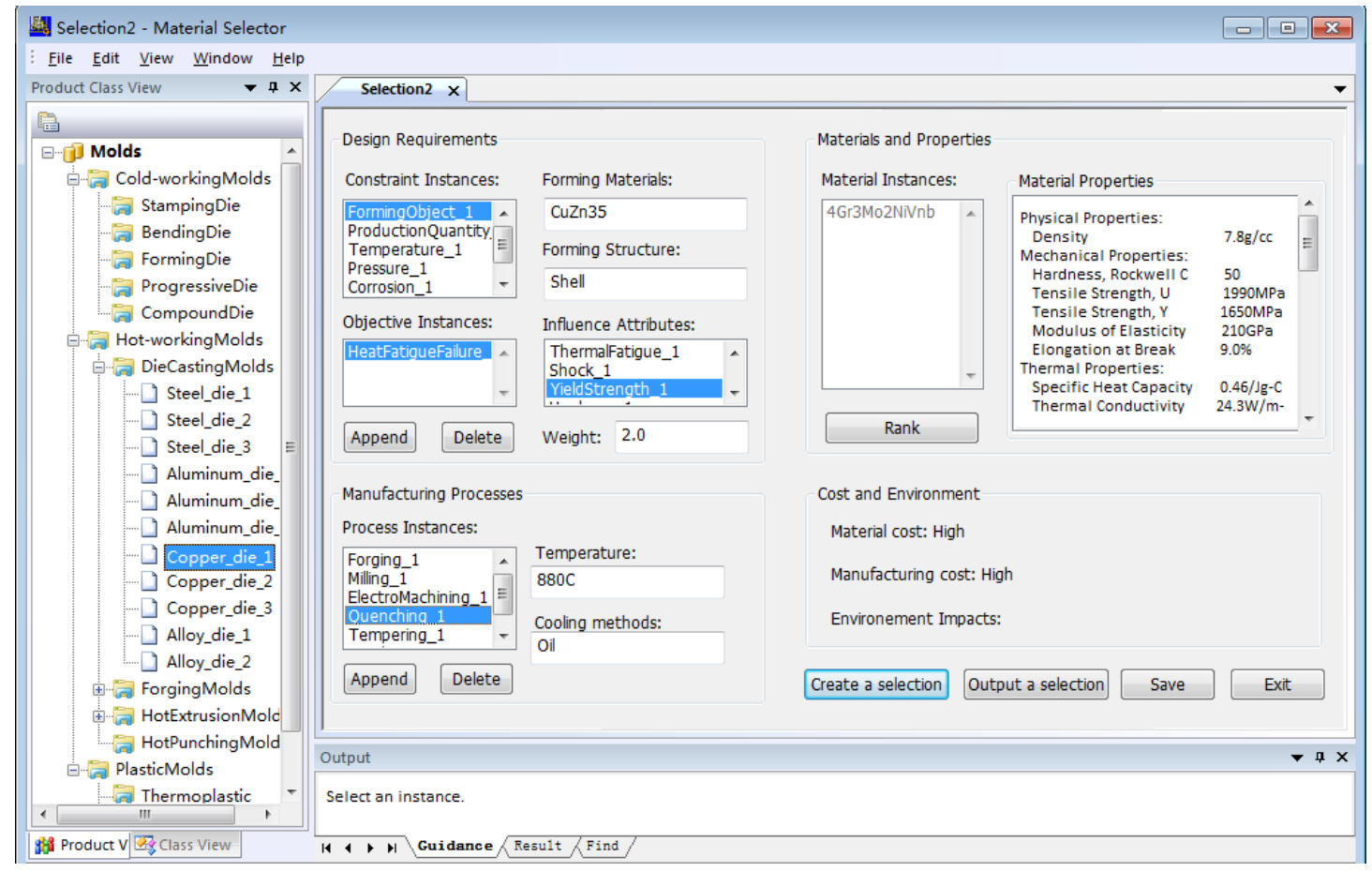

Fig. 13. User interface of the material selector

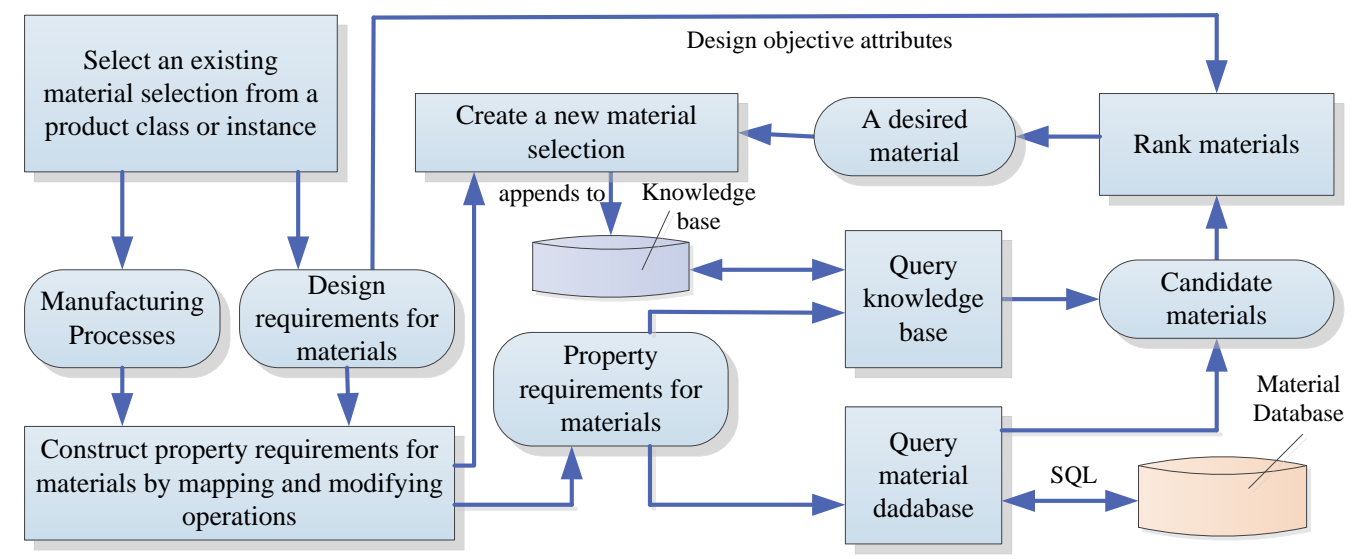

Fig. 14. Aninformation flowdiagram for creating a new material selection 